\title{
Propuesta metodológica en la aplicación de la derivada en Ingeniería Agroforestal, II semestre 2013
}

\author{
Ernesto Vanegas Sevilla ${ }^{1}$ \\ Yahaira Bermúdez Vargas ${ }^{2}$ \\ Luis Antonio López Mairena ${ }^{3}$
}

\section{Resumen}

Esta investigación ha evidenciado la importancia que tienen las estrategias metodológicas en el proceso educativo en las aplicaciones de las derivadas en la carrera de Ingeniería Agroforestal, pretendiendo que los discentes desarrollen autonomía para enriquecer su conocimiento y un aprendizaje significativo, forjar consciencia sobre la importancia que tiene la implementación de los softwares en las aplicaciones matemáticas.

El enfoque mixto permitió enriquecer los datos cuantitativos con la información cualitativa admitiendo la recolección, análisis y vinculación de los indicadores. La población fue conformada por 58 estudiantes de Ingeniería Agroforestal de los recintos de URACCAN Bluefields y Siuna (Las Minas), considerando como muestra a toda población, debido a que es muy pequeña al aplicar la fórmula triola para el cálculo de la muestra de una población finita con un margen de error del 1\% y un nivel de confianza del 95\%. El estudio determinó que la mayor dificultad es que los estudiantes no traen un dominio pleno de la Matemática (álgebra y geometría), razón por cual se les dificulta la comprensión y resolución de las derivadas.

Palabras clave: Aplicaciones de las derivadas; cálculo integral; cálculo diferencial; estrategias metodológicas; Ingeniería Agroforestal; resolución de las derivadas.

\section{Summary}

This research has demonstrated the importance of methodological strategies in the educational process related to the applications of by-product in the career of Agroforestry Engineering, with the pretension that students develop autonomy to enrich their knowledge and achieve meaningful learning, and also to build awareness about the importance of the implementation of software in the mathematics applications.

\footnotetext{
Licenciado en Biología Marina. Docente del Recinto Bluefields, evanegassevilla1o@gmail.com Licenciada en Informática Admón. Docente de Siuna. yacep82@yahoo.com

Tutor, Master en Docencia Universitaria, Coordinador Ing. Civil. antoniolopez95@gmail.com
} 
The mixed approach permitted to enrich the quantitative data with qualitative information admitting the collection, analysis and link with the indicators. The population was made up of 58 students of Agroforestry Engineering from URACCAN (Bluefields and Siuna Campus (Las Minas), considering the entire population as sample, due to the fact that it's very small when applying the triola formula to calculate the sample of a finite population, with a margin of error of $1 \%$ and a reliability level of $95 \%$. The study showed that the main difficulty is that students don't have a full domain of mathematics (algebra and geometry), and that's the reason why they have difficulty to understand and resolve the by-products.

Keywords: Application of by-products; integral calculus; differential calculus; methodological strategies; Agroforestry Engineering; resolution of the derivatives.

\section{Introducción}

La Matemática es de gran importancia para la vida diaria, ya que se aplica a cualquier campo; ingeniería, economía, biología, entre otras; sin embargo, a diario nos encontramos con dificultades en cuanto a su aprendizaje, sobre todo a nivel de Educación Superior. Por tanto, una alternativa para dinamizar la enseñanza y el aprendizaje de las matemáticas es aplicar un aprendizaje basado en problemas o situaciones problemáticas que permita a los estudiantes incursionar en éstas, desarrollando niveles amplios de participación, ya que ellos son los partícipes de su propio aprendizaje, en donde ponen en juego su saber previo y reorganizan con ayuda de sus compañeros y el docente, una red dinámica de relaciones conceptuales en función de la nueva información.

Por consiguiente, el cálculo es una de las áreas de las matemáticas que mayor dificultad aducen los educandos de nivel universitario en nuestro país. Es así que el aprendizaje de este y la comprensión de las aplicaciones de las derivadas significan un reto tanto para el docente como el educando, ya que trae consigo dificultades en cuanto al pensamiento numérico y abstracto se refiere. Artigue (1995) expresa que si bien muchos estudiantes pueden aprender a realizar de forma mecánica cálculos de derivadas, primitivas y resolver algunos problemas, se encuentran grandes dificultades para alcanzar una verdadera comprensión de los conceptos y su aplicación.

Mediante la realización de esta investigación se elaboró una propuesta metodológica donde se enfoca lo científico, teórico, técnicas, reglas, procedimientos, creatividad e innovación; permitiendo despertar en los estudiantes habilidades, destreza, interés y motivación en las resoluciones de ejercicios y problemas de derivadas. Esta propuesta facilitará el proceso enseñanza aprendizaje de las derivadas y sus aplicaciones, ya que será una herramienta didáctica para el docente y el estudiante, con el fin de mejorar la comprensión de la misma. 
Para la realización de este trabajo investigativo se propusieron las siguientes hipótesis:

"Las estrategias metodológicas utilizadas por los docentes y las estrategias de aprendizajes de los estudiantes tienen relación en el proceso enseñanza - aprendizaje de las derivadas y sus aplicaciones en el campo de la Ingeniería Agroforestal en el primer año de la carrera."

Ho = No existe relación entre las estrategias metodológicas con las estrategias de aprendizaje utilizadas en el proceso del aprendizaje de las derivadas.

H1= Existe una relación entre las estrategias metodológicas con las estrategias de aprendizaje utilizadas en el proceso del aprendizaje de las derivadas.

\section{Revisión de literatura}

La Matemática han significado un reto importante en el estudiantado, y en muchos casos es considerada un problema sobre todo a nivel universitario cuando se tienen dificultades con las matemáticas básicas.

Consecuente con estas dificultades que se presentan en el estudio de la Matemática los investigadores Lozano Y. (2011), Rojas, P. (2010) y Flores, W. (2012), realizaron trabajos investigativos en el área del cálculo específicamente basándose en las derivadas y sus aplicaciones al campo de estudio, determinando cada uno de ellos lo siguiente:

Flores, W. (2012), realizó la investigación titulada: "Estudio de dos metodologías utilizada en la enseñanza aprendizaje de la derivada en los estudiantes de Administración de Empresa: Influencia en el rendimiento de la asignatura Matemática Financiera", con el objetivo de analizar las metodologías que utilizan dos profesores de matemáticas en la enseñanza aprendizaje de la derivada con el estudiantado de Administración de Empresas en la Universidad de las Regiones Autónomas de la Costa Caribe Nicaragüense (URACCAN).

Los principales hallazgos de Flores manifiestan algunas carencias didácticas relacionadas con el contexto social donde se desarrollan los estudiantes y en cuanto a las estrategias de evaluación que realiza el profesor B, se inclina a una evaluación más al contenido matemático, mientras que el profesor $\mathrm{A}$, hace más hincapié en una evaluación aplicada, es decir, el conocimiento matemático resuelve problemas económicos que están en el contexto social de la carrera de Administración de Empresas.

Sin embargo, en las últimas décadas los estudios sobre la problemática de la enseñanza-aprendizaje de las matemáticas se han enriquecido considerablemente con la incorporación de variables de tipos afectivos y socioculturales. Estas perspectivas no 
niegan la naturaleza cognitiva del aprendizaje matemático, pero señalan que el factor cognitivo no es el único participante en el aprendizaje, pues es un proceso compartido con el mundo afectivo y el contexto del sujeto que aprende.

\section{Estrategias de aprendizaje}

En cuanto a estrategias de aprendizajes Duval (1998) expresa que el uso de distintas representaciones es esencial en el desarrollo del pensamiento y en la producción del conocimiento. Distintos autores apoyan esta idea y manifiestan que llegar a comprender un concepto matemático implica realizar procesos de conversión entre diferentes registros de representación, manifestados por la posibilidad de movilización y de articulación entre los mismos (Rico, 2000, D’ Amore, 2002).

Artigue (1995) expresa que si bien muchos estudiantes pueden aprender a realizar de forma mecánica cálculos de derivadas, primitivas y resolver algunos problemas, se encuentran grandes dificultades para alcanzar una verdadera comprensión de los conceptos involucrados y un desarrollo adecuado de métodos de pensamiento que son el centro de este campo de la matemática. Un fenómeno educativo de la matemática es el predominio de métodos algebraicos y algorítmicos. Cantoral y Mirón (2000), señala que esto provoca que una gran cantidad de alumnos no logren dar sentido y significado a los conceptos básicos, de modo que, aun siendo capaces de derivar una función, no pueden reconocer en cierto problema la necesidad de una derivación.

Un aprendizaje es relevante cuando el estudiantado concibe la utilidad y lo puede aplicar. Es común que en las escuelas se invierta tiempo en contenidos que los mismos niños y niñas cuestionan: ¿Para qué estamos estudiando esto? ¿Para qué nos va a servir? Y por más que busquen, no logren encontrarle sentido a lo que están estudiando. Lo mismo sucede con el contenido que los libros de texto y otros materiales presentan, donde la temática y las actividades que se incluyen carecen de relevancia (Martínez, 2004, pág. 61).

El Aprendizaje Basado en Problemas (ABP) es uno de los métodos de enseñanzaaprendizaje que ha tomado más arraigo en las instituciones de Educación Superior en los últimos años. El ABP es una metodología centrada en el aprendizaje, en la investigación y reflexión que siguen los discentes para llegar a una solución ante un problema planteado por el docente.

Barrows (1986), define al ABP como: "un método de aprendizaje basado en el principio de usar problemas como punto de partida para la adquisición e integración de los nuevos conocimientos". En esta metodología los protagonistas del aprendizaje son los propios alumnos, que asumen la responsabilidad de ser parte activa en el proceso. 
Por consiguiente en el 2006, Prieto defendiendo el enfoque de aprendizaje activo en donde señala que "el aprendizaje basado en problemas representa una estrategia eficaz y flexible que, a partir de lo que hacen los estudiantes, puede mejorar la calidad de su aprendizaje universitario en aspectos muy diversos". Así, el ABP ayuda al alumno a desarrollar y a trabajar diversas competencias.

\section{Utilidad de la tecnología en la enseñanza y aplicaciones de las derivadas}

En este sentido Martínez (2003), dice que las nuevas tecnologías precisan de unas necesidades previas, sin las cuales no puede hablarse de su incorporación a ningún ámbito de la enseñanza entre ellas:

El acceso técnico: Tiene que ver con la posibilidad material de disponer de acceso a estas tecnologías a los medios y servicios que proporcionan.

El acceso práctico: Se relaciona con la disponibilidad del tiempo necesario para el empleo de las tecnologías, al igual que con preparar el proceso de su uso como soporte para la enseñanza y como medio para el aprendizaje.

El acceso operativo: Referido a los conocimientos que van a permitir el manejo de la herramienta tecnológica.

El acceso criterial: La utilización de las tecnologías precisa de una actitud previa crítica con la propia tecnología y que facilita la toma de decisiones sobre su utilización. La posibilidad de responder a la pregunta de por qué esta tecnología aquí y ahora es una cuestión fundamental.

El acceso relacional científico tecnológico: Vinculado con los requisitos previos que necesitan tener del proceso de enseñanza en que se pretende incidir con las tecnologías.

Además Miratía (2005), sugiere que la aplicación de las tecnología de la información y comunicación en la educación superior, exige que las y los docentes dominen su uso en los procesos de enseñanza-aprendizaje y que posea los conocimientos mínimos que le permita integrar y operar eficientemente con creatividad y autonomía estas herramientas tecnológicas como un recurso más en las áreas de desarrollo del currículo.

Por otra parte, que sea capaz de evaluar el software educativo, los multimedios e internet para apoyar las actividades de aprendizaje en la construcción de nuevos conocimientos y determinar la forma y el momento oportuno para la integración de las Tic's en su práctica pedagógica. 


\section{Materiales y métodos}

Este estudio se realizó en la URACCAN en los recintos de Bluefields y Siuna a los estudiantes del primer año de la carrera de Ingeniería Agroforestal, tiene un enfoque mixto, un alcance correlacional y de corte transversal, la población es de 58 estudiantes y dos docentes, la muestra representativa fue de 58 estudiantes, la cual se calculó con un margen de error del 1\% y un nivel de confianza del 95\%, a la cual se le aplicó: encuestas, entrevistas, test de evaluaciones y grupos focales para recolección de datos y luego procesados con los programas en IBM SPSS Statistics para el análisis cuantitativo y para el análisis de la información cualitativa se utilizó el modelo de redes sistémicas para hacer comparaciones sobre los resultados encontrados en la investigación.

\section{Resultados y discusión}

La percepción de los estudiantes sobre las aplicaciones de las derivadas se distribuyó en tres categorías: percepción negativa, media y positiva. El 79.31\% de los estudiantes tienen una percepción positiva, en la cual opinaron que las matemáticas son importantes, interesantes y que son útiles en la carrera de ingeniería Agroforestal, ya que tienen aplicaciones al campo laboral (mediciones, capacidades máximas, mínimas de un terreno), esto permite afirmar que el estudio de las aplicaciones de las derivadas estimulan los sentidos, despertando en ellos motivación para aprender lo que le es necesario para la resolución de problemas o situaciones que enfrenta un ingeniero en su profesión.

Mientras que $18.97 \%$ de los estudiantes tienen una percepción media, debido a que no han logrado relacionar los conocimientos teóricos de las derivadas con el quehacer profesional de su carrera y 1.72\% tiene una percepción negativa, ya que no recibieron clases constantemente, lo cual no les permitió tener una claridad sobre sus aplicaciones de las derivadas en su carrera (Cuadro No. 1).

\begin{tabular}{|c|c|c|c|c|}
\hline \multicolumn{5}{|c|}{ Cuadro No. 1: Relación Sexo - Percepción } \\
\hline & \multicolumn{3}{|c|}{ Percepción } & \\
\hline Sexo & Negativa & Media & Positiva & Total \\
\hline Masculino & o & 6 & 23 & 29 \\
\hline Femenino & 1 & 5 & 23 & 29 \\
\hline Total & 1 & 11 & 46 & 58 \\
\hline Porcentajes & 1.72 & 18.97 & 79.31 & 100 \\
\hline
\end{tabular}


Del mismo modo se refleja a través de las medidas de tendencia central que los estudiantes tienen una percepción positiva, ya que el estudiantado en promedio se ubican en la escala Likert en 3.8 y el 50\% está por encima del valor de 4.0 y el restante $50 \%$ por debajo de este valor. (Cuadro No.2)

\begin{tabular}{|l|l|r|}
\hline \multicolumn{2}{|c|}{ Cuadro No. 2: Descriptivos } \\
\hline \multicolumn{2}{|c|}{ Variable: Percepción } & Estadístico \\
\hline Media & 3.8345 \\
\hline $\begin{array}{l}\text { Intervalo de confianza } \\
\text { para la media al 95\% }\end{array}$ & Límite inferior & 3.6239 \\
\cline { 2 - 3 } & Límite superior & 4.0451 \\
\hline Mediana & 4 \\
\hline Varianza & 0.642 \\
\hline Desviación Típica & 0.801 \\
\hline
\end{tabular}

Asimismo, los docentes "A" y "B" confirmaron que la percepción juega un papel importante en la educación, porque les permite estimular los sentidos en los estudiantes, estimulando una motivación e interés por el estudio de las aplicaciones de las derivadas aplicada en la carrera de Ingeniería Agroforestal, permitiendo la resolución de problemas de producción y determinación de áreas (red sistémica \#1).

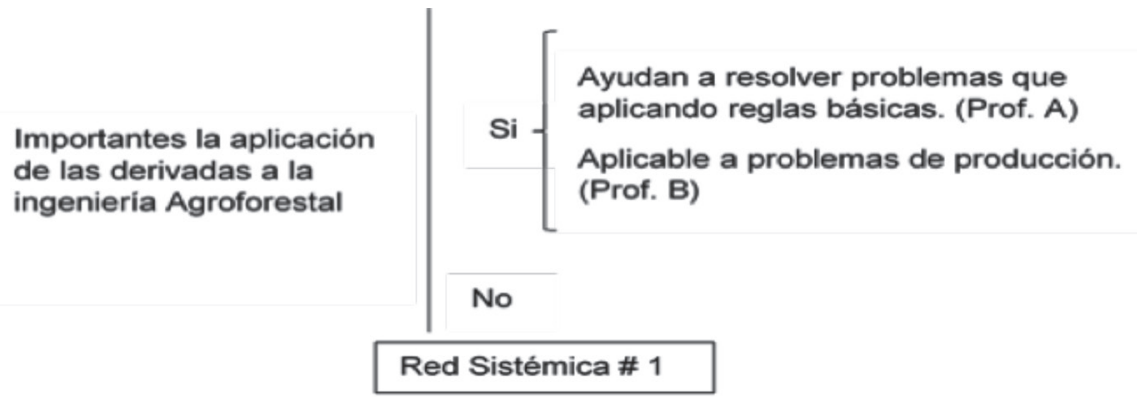

Por consiguiente, las opiniones de los estudiantes y docentes demuestran una percepción positiva sobre el estudio de las aplicaciones de las derivadas, lo cual se relaciona con la teoría de Neisser citado por Bayo (1987), donde establece que la percepción es importante en el proceso de enseñanza aprendizaje en cualquier disciplina, ya que permite desarrollar un conjunto de procesos y actividades relacionados con la estimulación que alcanza a los sentidos, mediante los cuales el docente provee de información al estudiante tomando en consideración el entorno y el uso de los contenidos en el campo laboral. Del mismo modo con el planteamiento de Gibson (1974), que el organismo sólo percibe aquello que puede aprender y le es necesario para sobrevivir. 


\section{Métodos y estrategias que utilizan los docentes en el proceso enseñanza de las aplicaciones de las derivadas}

Los docentes "A" $\mathrm{y}$ "B" en el proceso de la enseñanza aprendizaje de las aplicaciones de las derivadas utilizaron tres métodos: Expositivo, aprendizaje basado en resolución de problemas y métodos de casos. Estos métodos permitieron que los estudiantes de manera individual o colectiva analizaran e interpretaran situaciones vinculadas al campo laboral. Además, el aprendizaje basado en resolución de problemas les permitió a los estudiantes ser protagonista de sus propios aprendizajes, asumiendo la responsabilidad de ser parte activa en el proceso educativo, con el fin de tomar decisiones sobre el problema en estudio. Estas valoraciones que hacen los docentes son sustentadas en las teorías planteadas por los autores Prieto (2006) y Barrows (1986), en donde mencionan que es eficaz y flexible, para que los estudiantes puedan mejorar la calidad de su aprendizaje universitario desarrollando diversas competencias.

Así mismo los docentes mencionaron el uso de diferentes estrategias, de las cuales las más efectivas fueron: definición de la temática, presentación de fórmulas o tabla de las derivadas, identificando las diferencias entre cada una de las reglas de las derivadas, ejercicios acorde a la fórmula que se va desarrollando, ejercicios de máximos y mínimos, trabajos individuales o colectivos que se ilustran en la red sistémica \# 2. En dichas estrategias los docentes utilizaron los siguientes materiales didácticos: libros, Data show, videos, folletos, cinta métrica, papelones y la pizarra, esto les permitió que el estudiantado se motivara por el estudio de la misma. Díaz, F. y Hernández G (1999), consideran que estos recursos motivan a los universitarios y fomentan en ellos el interés por el estudio de las aplicaciones de las derivadas.

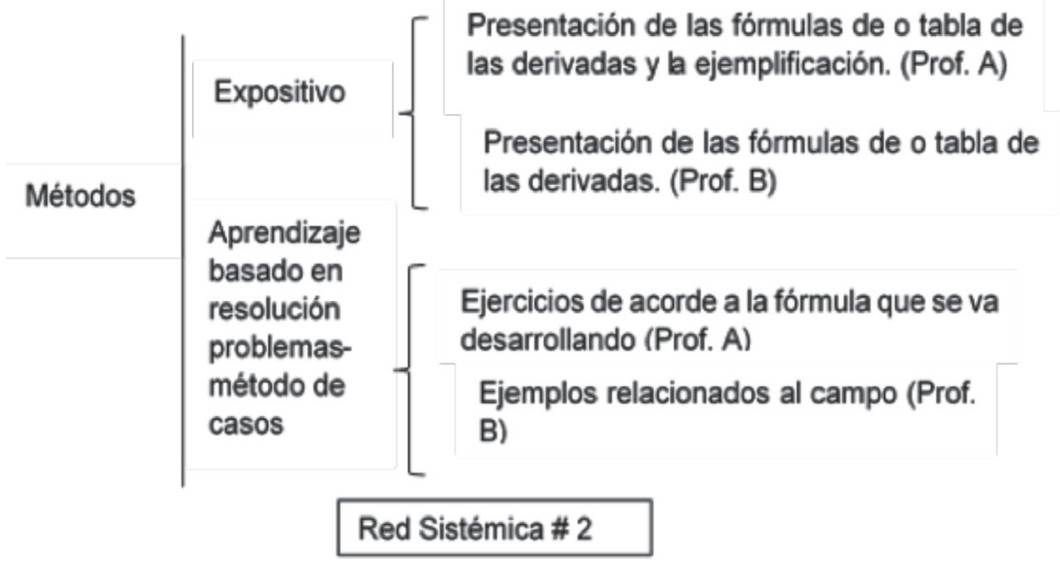

Análogamente, el 70.37\% de los estudiantes consideran que los docentes aplican estrategias buenas, ya que tienen dominio de las derivadas, aplican las reglas de las derivadas, orientan trabajos individuales o en grupos, asignan investigaciones para posteriormente hacer plenario, resolución de ejercicios en la pizarra relacionándolo 
al campo de la Ingeniería Agroforestal (aplicabilidad a la topografía y productividad), estas estrategias contribuyen al proceso enseñanza aprendizaje de las aplicaciones de las derivadas, además se ilustran en la red sistémica \# 3 las estrategias que utilizaron los grupo A y B. Cabe agregar que el 29.63\% opinaron que las estrategias aplicada son regulares.

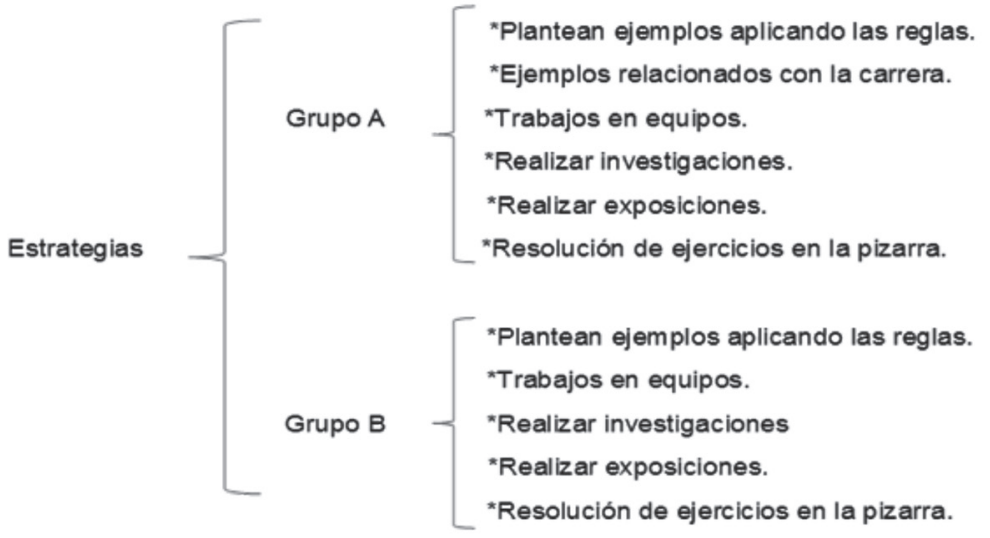

Red sistémica \# 3

Del mismo modo, las medidas de tendencia central reflejan que los estudiantes en promedio se ubican en la escala Likert en 3.5 reflejando que los docentes aplican buenas estrategias en el proceso enseñanza aprendizaje de las aplicaciones de las derivadas y que el 50\% está por encima del valor de 3.75 y el restante $50 \%$ por debajo de este valor (Cuadro No. 3), esto permite afirmar lo ante mencionado por los estudiantes.

\begin{tabular}{|l|l|r|}
\hline \multicolumn{2}{|c|}{ Cuadro No. 3: Descriptivos } \\
\hline \multicolumn{2}{|c|}{ Variable: Estrategias de enseñanza } & Estadístico \\
\hline Media & 3.5556 \\
\hline $\begin{array}{l}\text { Intervalo de confianza } \\
\text { para la media al 95\% }\end{array}$ & Límite inferior & 3.3593 \\
\cline { 2 - 3 } & Límite superior & 3.7518 \\
\hline Mediana & 3.75 \\
\hline Varianza & 0.517 \\
\hline Desviación Típica & 0.71887 \\
\hline
\end{tabular}

\section{Dificultades en la enseñanza aprendizaje de las aplicaciones de las derivadas}

Los docentes a través de la encuesta mencionaron que existen dificultades en la enseñanza aprendizaje de las derivadas, ya que los estudiantes tienen poco conocimientos básico de las matemáticas y esto es la base para el cálculo, por lo tanto los estudiantes no asimilaron en su totalidad los contenidos desarrollados. Esta debilidad ocasionó en 
los estudiantes poco interés por la asignatura debido a que se les dificultaba resolver los ejercicios y problemas de derivadas aplicado al campo agroforestal. De la misma manera se les consultó a los estudiante manifestando que ellos tienen una actitud positiva hacia la asignatura del cálculo, sin embargo se desmotivan cuando no lograban resolver los ejercicios y problemas asignado por el docente, debido a que no tenían una buena base en la matemática básica, ya que provienen de modalidades nocturnas y a distancia, en estas modalidades no recibieron clases de matemática regularmente por diversas afectaciones en los cursos lectivos de secundarias. En consecuencia a esta limitante los estudiantes no logran solucionar correctamente los diferentes problemas, ya que deben buscar, entender e integrar los conceptos básicos de la asignatura.

\section{Estrategias de aprendizajes de los estudiantes}

A través de la opinión de los estudiantes se categorizaron las estrategias de aprendizaje de tres maneras: estrategias positivas, regulares y negativas, la cual se observa en el figura no. 1. Según estas categorías el $64.7 \%$ utilizan estrategias positivas para su aprendizaje, de las cuales son las siguientes: atiende las explicaciones del docente en el desarrollo de las clases, realizan preguntas para quedar claro sobre el tema en estudio, participan en la resolución de ejercicios o problemas en la pizarra, realizan autoestudio, participan en círculos de estudios, realizan investigaciones y consultan al docente sobre algunas dificultades que tienen en la resolución de ejercicios o problemas. Cabe agregar que estas mismas estrategias fueron enunciadas en los grupos focales A y B. Además los estudiantes consideran que estas actividades les han permitido mejorar su aprendizaje de las derivadas y sus aplicaciones.

Al mismo tiempo el $35.3 \%$ de los estudiantes manifestaron que no utilizan en su totalidad todas las estrategias antes mencionadas, por lo tanto consideraron que sus estrategias son regulares, ya que participan poco en las resoluciones de ejercicios o problemas en la pizarra, a veces realizan círculos de estudios y no consultan con los docentes sobre el tema en estudio.

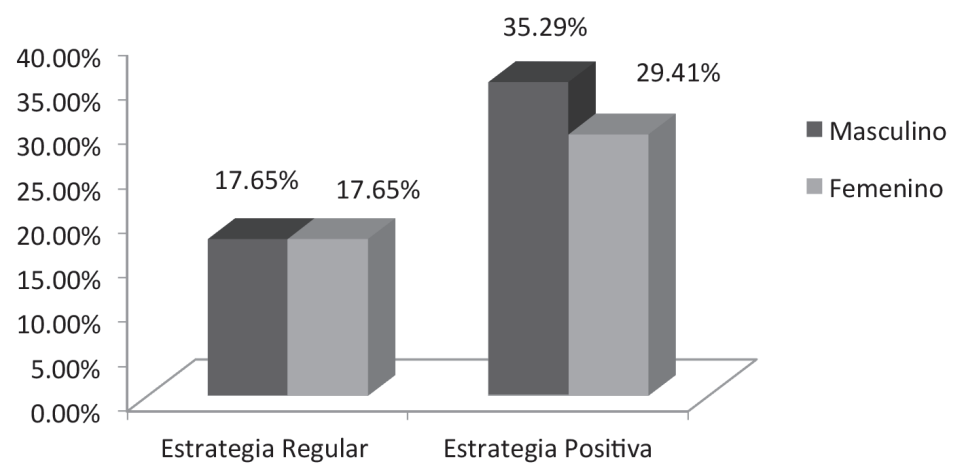

Figura No. 1. Porcentaje de estrategias de aprendizaje en los estudiantes 
De la misma manera, las medidas de tendencia central reflejan que los estudiantes en promedio, se ubican en la escala Likert en 3.4 y que el 50\% está por encima del valor de $3.5 \mathrm{y}$ el otro $50 \%$ por debajo de este valor. Estos valores manifiestan que los estudiantes utilizan estrategias positivas en el proceso de aprendizajes de las derivadas y sus aplicaciones. La cual se ilustran en la cuadro No. 4.

\begin{tabular}{|l|l|r|}
\hline \multicolumn{2}{|c|}{ Cuadro No. 4: Descriptivos } \\
\hline \multicolumn{2}{|c|}{ Variable: Estrategias de Aprendizajes } & Estadístico \\
\hline Media & 3.4412 \\
\hline $\begin{array}{l}\text { Intervalo de confianza } \\
\text { para la media al 95\% }\end{array}$ & Límite inferior & 3.2266 \\
\cline { 2 - 3 } & Límite superior & 3.6558 \\
\hline Mediana & 3.5 \\
\hline Varianza & 0.582 \\
\hline Desviación Típica & 0.7629 \\
\hline
\end{tabular}

Con referencia a lo antes mencionado, nos permite afirmar que las actividades que utilizan los estudiantes son fundamentales para el aprendizaje, ya que mejora la comprensión, análisis e interpretación de las derivadas en el campo de la Ingeniería Agroforestal.

De la misma manera, lo plantea Jean Díaz (citado por Anijovich R y Mora S, 2009) expresando que las actividades "son instrumentos para crear situaciones y abordar contenidos que permiten al estudiante vivir experiencias necesarias para su propia transformación". Este planteamiento concuerda con las estrategias utilizadas por los estudiantes, ya que estas herramientas les ayudan a estructurar las experiencias de aprendizaje permitiéndole apropiarse de diferentes saberes. Además permite crear condiciones apropiadas para que los estudiantes construyan aprendizajes con sentido, es decir, conocimientos que estén disponibles para ser utilizados de manera adecuada y flexible en situaciones variadas.

\section{Factores que inciden en el proceso de aprendizaje}

Los factores que incidieron en el aprendizaje de las derivadas y sus aplicaciones fueron: poco dominio de la matemática básica, no dedicaron el tiempo necesario para afianzar sus conocimientos, debido a esto no les permite al estudiante desarrollar el razonamiento abstracto y pensamiento deductivo, ya que son la base para los conocimientos subsiguientes.

Además los estudiantes manifestaron que son pocos los problemas vinculados a la carrera, por lo tanto no le encuentran la relación en su totalidad para llevarlo a la práctica, por tal razón consideran que las matemáticas y el cálculo son difíciles de comprender y aplicar. 


\section{Importancia de una propuesta metodológica}

Los docentes consideran que una propuesta metodológica es una herramienta que permitirá mejorar el proceso enseñanza aprendizaje de las derivadas y sus aplicaciones, ya que los libros de cálculo de los diferentes autores no cuentan con suficientes problemas relacionados al campo de la Ingeniería Agroforestal. Asimismo consideran que debe ser una propuesta contextualizada a la carrera en estudio.

Además esta propuesta mejoraría el desarrollo de las clases, motivando a los estudiantes para el estudio de la misma, a través de las diversas situaciones o problemas vinculado a su carrera.

Cabe mencionar que los docentes consideran que las aplicaciones más utilizadas en el campo de la Ingeniería Agroforestal es el cálculo de máximo y mínimo, haciendo uso de la primera y segunda derivada, por lo tanto en la propuesta debe elaborarse tomando en cuenta estos contenidos, al igual que la parte de la interpretación geométrica al momento de determinar área (red sistémica No. 4).

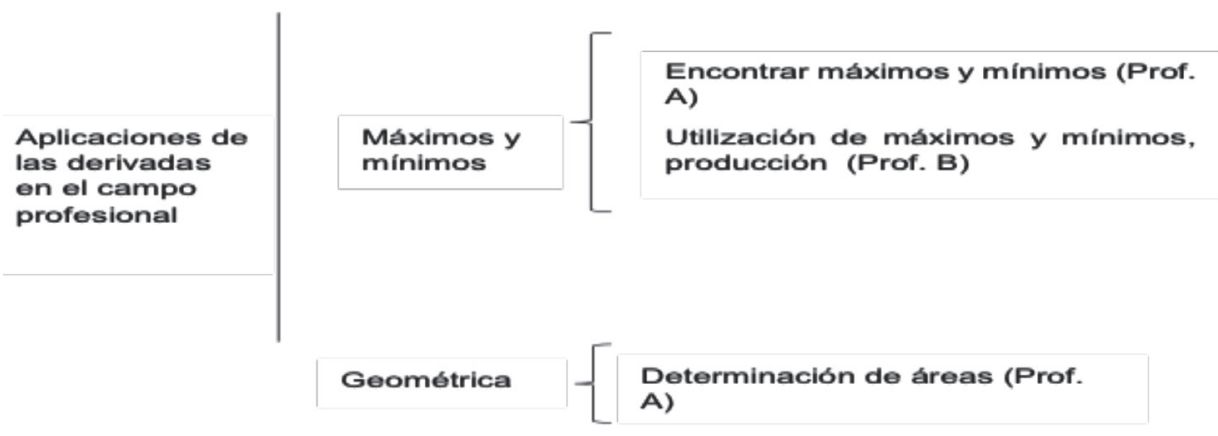

Red Sistémica \#4

\section{Utilización de software para la resolución de ejercicios}

Los docentes manifestaron que no hacen uso de software como estrategias de enseñanza, ya que no cuentan con los medios necesarios para su implementación con los estudiantes, tales como los softwares y equipos necesarios para su aplicación, pero consideran que es importante su utilidad para que los estudiantes verifiquen sus respuestas, con el fin de promover su interés y motivación para la resolución de la misma (red sistémica \# 5). De la misma manera, lo señala Martínez (2003), dice que las nuevas tecnologías precisan de unas necesidades previas, sin las cuales no puede hablarse de su incorporación a ningún ámbito de la enseñanza. Estas son: El acceso técnico, acceso práctico, acceso operativo, acceso criterial y el acceso relacional científico tecnológico. 


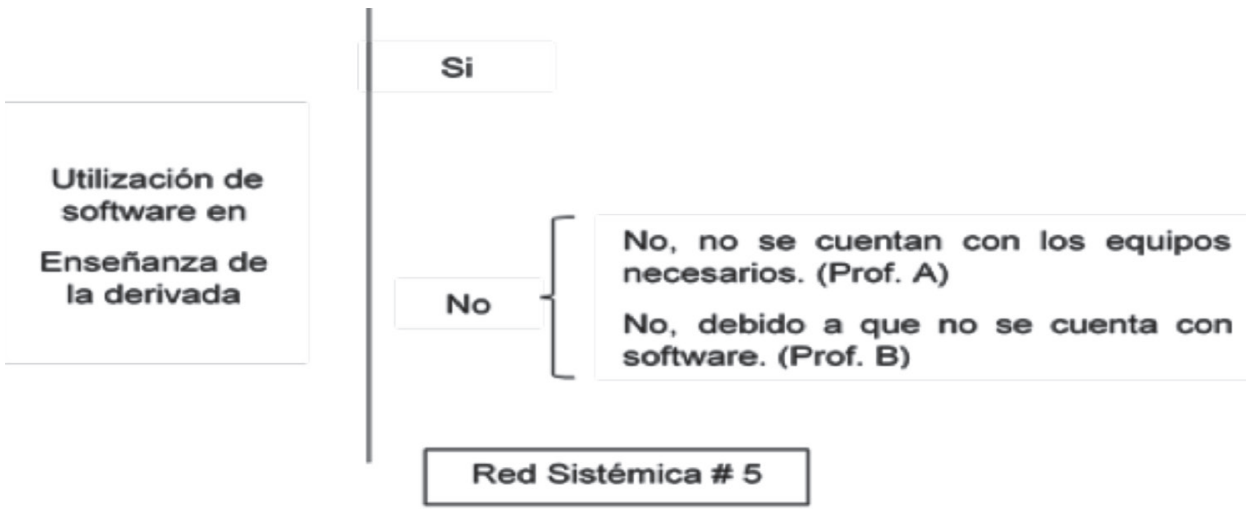

Además hacen mención que las utilizaciones de instrumentos modernos son necesario en el proceso enseñanza aprendizaje, considerando que algunos de los instrumentos que se pueden utilizar son los GPS y Teodolitos, ya que a través de ellos se pueden realizar mediciones y ubicación de coordenadas, las cuales se pueden utilizar para el cálculo de máximo o mínimo de un área determinada y de esa forma poder lograr que los estudiantes asimilen mejor la aplicación de las derivadas en ejercicios muy utilizados en su carrera profesional.(Red sistémica No. 6)

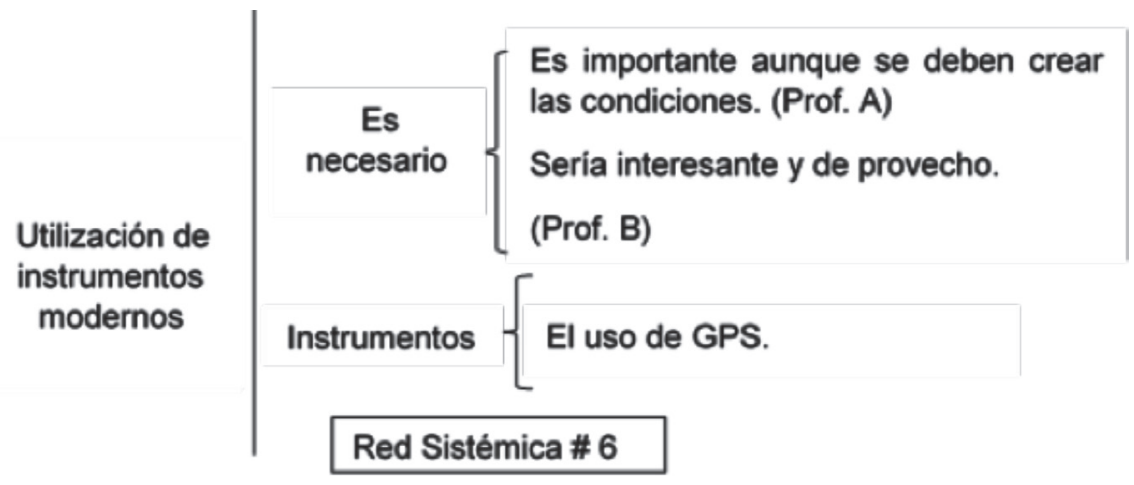

Por lo tanto, es interesante y de mucho provecho tanto para el docente como al estudiantado, siempre y cuando se faciliten los equipo necesario para lograr un aprendizaje significativo de las derivadas y las aplicaciones al campo agroforestal. Ambos docentes están en total acuerdo de la importancia y ventajas que implicaría la utilización de softwares y equipos para afianzar los conocimientos teórico y relacionarlo con el quehacer de un profesional agroforestal, pero esto se logrará siempre y cuando se crean las condiciones apropiada para el uso de estas estrategias de enseñanza.

\section{Conclusiones}

Al realizar esta investigación se manifestaron diversas opiniones de estudiantes y docentes a través de las encuestas, grupos focales, entrevistas, observación indirecta 
sobre la metodología y estrategias de enseñanza aprendizaje de las aplicaciones de las derivadas en su contexto, después del análisis podemos concluir los siguientes:

Existe evidencia estadísticamente significativa para rechazar la hipótesis nula (No existe relación entre las estrategias de enseñanza y la estrategias de aprendizaje en las aplicaciones de las derivadas), por tanto podemos afirmar que existe relación entre las estrategias metodológicas utilizadas por los docentes y las estrategias de aprendizajes utilizadas por los estudiantes, para el aprendizaje de las aplicaciones de las derivadas en el campo laboral de la carrera de Ingeniería Agroforestal.

El 79.31\% de los estudiantes tienen una percepción positiva sobre la utilidad de las aplicaciones de las derivadas en el campo de la Ingeniería Agroforestal, ya que a través de ellas se pueden calcular mediciones, capacidades, áreas máxima y mínima de un terreno.

Los estudiantes demuestran una actitud positiva hacia el estudio de las aplicaciones de las derivadas y su importancia e utilidad al campo agroforestal, ya que esto les permitirá aprender nuevas técnicas para luego aplicarla en el quehacer de un profesional.

Los docentes emplean la metodología "Aprendizaje Basado en Problema", pero consideran que los libros de cálculo no contienen muchos problemas relacionado con la carrera agroforestal, lo cual se les dificultad la enseñanza de la misma.

Los docentes utilizan estrategias variadas de enseñanza en el desarrollo del contenido, permitiendo mejorar la calidad de aprendizaje de los universitarios, con el fin de desarrollar en ellos habilidades y destreza en las resoluciones de problemas vinculado a la carrera.

Los estudiantes manifestaron que las estrategias que utilizan los docentes son fundamentales en el proceso enseñanza aprendizajes de las derivadas y sus aplicaciones, ya que les permite afianzar los conocimientos.

Los estudiantes del grupo A y B realizan autoestudio, círculo de estudio y consultas al docente, para afianzan el conocimiento en la resolución de problemas aplicado al campo laboral, pero consideran que el problema radica en la mala base que traen de la matemática básica. 


\section{Lista de referencias}

Artigue, M. (1995). La enseñanza del principio del cálculo: problemas epistemológicos, cognitivos y didácticos. Grupo Editorial Iberoamérica. Bogotá, Colombia.

Barrows, H.S. (1986). A Taxonomy of problem-based learning methods, en Medical Education, 20/6, 481-486.

Cantoral, R. \& Farfán, R. (2003). Mathematics education: a vision of its evolution. Educational Studies in Mathematics 53 (3), 255-270

Cantoral, R. \& Mirón, H. (200o). Sobre el estatus de la noción de derivada: de la epistemología de Joseph Louis La Grange al diseño de una situación didáctica. Revista Latinoamericana de Investigación en Matemática Educativa. 3(3), pp. 265-292.

Díaz B., F. y Hernández R., G. (1999). Estrategias docentes para un aprendizaje significativo. McGraw Hill, México, 232p.

Danilov, M.A, Skatkin, M. N. Didáctica de la Escuela Media. Editorial Pueblo y Educación. 1988.

DANILOV, M. A Didáctica de la escuela media/. Editorial de libros para la educación, 1980.

Danilov M. A. y M. N. Skatkin, (1980). Didáctica de la escuela media. Editorial Libros para la educación. C de La Habana. Pág. 123.

Duval, R. (1998). Registros de representación semiótica y funcionamiento cognitivo del Pensamiento. En HITT, F. (Ed.). Investigaciones en Matemática Educativa II. pp. 173-201. Grupo Editorial Iberoamérica: México. Traducción de: Registres de représentation sémiotique et functionnement cognitif de la pensée. Annales de Didactique et de Sciences Cognitives. Vol. 5 (1993)

Flores, W. O. (2011-12). Estudio de dos metodologías utilizadas en la enseñanza aprendizaje de la derivada a estudiantes de Administración de Empresa: Influencia en el rendimiento de la asignatura "matemática financiera". (Tesis de maestría). Universidad de Santiago de Compostela. Santiago de Compostela.

Gibson, James J. (1974). La percepción Del Mundo Visual. Editorial Infinito, Buenos Aires, Argentina. 
Lozano, Y. A. (2011). Desarrollo del concepto de la Derivada sin la noción del límite. Bogotá.

Martínez, F. (2003). El profesorado ante las nuevas tecnologías. En J. Cabero, F. Martínez y J. Salinas (Coords.), Medios y herramientas de comunicación para la educación universitaria (pp. 207-222)

Martínez, F.R. (2004). Nuevo Paradigma Educativo y Visión Integral del Aprendizaje. Guatemala.

Miratía, O. (2005). Efecto que Tiene en el Desempeño y Rendimiento de Estudiantes Universitarios la Implementación de un Curso de Computación a Distancia Bajo una Metodología Instruccional Basada en Web.

Prieto, L. (2006). Aprendizaje activo en el aula universitaria: el caso del aprendizaje basado en problemas, en Miscelánea Comillas. Revista de Ciencias Humanas y Sociales Vol.64. Núm.124. Págs. 173-196.

Rico, L. (2000). Sobre las nociones de representación y comprensión en la investigación en Educación Matemática. IV Simposio SEIEM. Huelva. España.

Rojas, P. (2010). El aprendizaje basado en problemas (ABP) como estrategia metodológica de enseñanza y aprendizaje de la integral indefinida en paralelo con derivadas y su incidencia en el rendimiento académico de los estudiantes de ingeniería en informática de INACAP, Chillán. (Tesis de maestría). Universidad tecnológica de Chile. Chillán. 